\title{
NEW EXACT RELATIONS FOR STEADY IRROTATIONAL TWO-DIMENSIONAL GRAVITY AND CAPILLARY SURFACE WAVES
}

\author{
DIDIER CLAMOND
}

\begin{abstract}
Steady two-dimensional surface capillary-gravity waves in irrotational motion are considered on constant depth. By exploiting the holomorphic properties in the physical plane and introducing some transformations of the boundary conditions at the free surface, new exact relations and equations for the free surface only are derived. In particular, a physical plane counterpart of the Babenko equation is obtained.
\end{abstract}

\section{INTRODUCTION}

Although studied for a long time [19], steady surface gravity and gravity-capillary waves remain a subject of active mathematical research and new solutions are still discovered [13, 23. Open questions concern not only extreme gravity waves and 'exotic' capillarygravity waves, but also regular gravity waves of finite amplitudes. Indeed, for the latter, many of their 'obvious' characteristics well-known to physicists remain to be rigorously proven mathematically [15, 17, 38]. In order to predict what features are likely true about steady irrotational gravity waves, some numerical evidences on the velocity, acceleration and pressure fields were given in 88. The present paper is somewhat a continuation of that work, providing new exact relations that could be useful for accurate computations, for new mathematical proofs and for proving already known results but with weaker assumptions.

In most mathematical and numerical investigations of two-dimensional surface waves, the problem is treated using conformal mapping. This approach, originally due to Stokes [37], is appealing because the fluid domain (whose shape is unknown a priori) is mapped into a known simple domain (e.g., strip, disk). Thus, in the conformal plane, the equations for steady waves can be reduced into a single equation written on a known line. Various equations have then been obtained as integral and pseudo-differential equations, such as the Babenko [1] and Nekrasov [29] equations (see [30, 42] for reviews). Conformal mapping can also be used in presence of vorticity; see [18] for recent results and conjectures.

Using conformal mapping is certainly the best approach for computing waves of small or finite amplitudes, but it is not so useful for extreme waves. This can be understood, for example, considering the limiting gravity wave with a $120^{\circ}$ inner angle at the crest. Such a wave has a power $2 / 3$ singularity at the crest in the conformal plane and, therefore, its $n$-th Fourier coefficient decays like $n^{-5 / 3}$ as $n \rightarrow \infty$. Conversely, the same wave in the physical plane has a discontinuous, but finite, free surface slope. Therefore, its $n$-th Fourier coefficient decays like $n^{-2}$ as $n \rightarrow \infty$. (A discussion on the Fourier coefficients of the Stokes waves, including the highest one, is given in [32].) Another example is Stokes' small 
parameter expansion for waves that are not too steep. It was shown in [7, 21] that these expansions have a better rate of convergence in the physical plane than in the conformal plane. The advantage of a physical plane formulation was also noticed for an accurate numerical resolution [22] and in the derivation of simple accurate analytic approximations [4, 5, 33]. A goal of the present paper is to advocate further the benefit of working in the physical plane instead of the conformal one.

In order to derive relatively simple exact equations in the physical plane, we follow the strategy used in [9, 11] for the surface reconstruction from bottom pressure measurements. In doing so, we obtain equations involving only the free surface (i.e., without velocity and pressure fields) that have nice features for analytical and numerical investigations. For instance, no derivatives need to be computed for gravity waves and only first-order derivatives are involved if surface tensions are included.

The paper is organised as follow. In section 2, we introduce the notations and precise definitions of the problem. The problem is then reformulated in a more tractable form in section 3. This reformulation involves a holomorphic function $\mathfrak{Q}$ that, for periodic waves, is determined in terms of a Fourier expansion in section 4. In this section, this Fourier series is also summed to obtain a Poisson-like integral formula. The definition of the Fourier coefficients, when applied at the free surface, provides a system of equations for the free surface, as demonstrated in section 5. In section 6, integral equations for the free surface are also derived. In particular, a physical plane counterpart of the Babenko equation is obtained. A regularisation of the integral equation for the free surface is given in the section 7, which should be suitable for numerical computations. Finally, a summary and some perspectives are given in section 8 .

\section{Definitions AND NOTATIONS}

Steady two-dimensional potential flows due to surface capillary-gravity waves in water of constant depth are considered. The fluid is homogeneous of with a constant density $\rho>0$. The pressure is denoted $P$ and, at the impermeable free surface, it is equal to the surface tension plus the constant atmospheric pressure $P_{\text {atm }} ; p \stackrel{\text { def }}{=}\left(P-P_{\text {atm }}\right) / \rho$ denotes the relative scaled pressure. The seabed is fixed, horizontal and impermeable.

Let $(x, y)$ be a Cartesian coordinate system moving with the wave, $x$ being the horizontal coordinate and $y$ the upward vertical one. The wave is $(2 \pi / k)$-periodic $(k=0$ for aperiodic waves) and $x=0$ is the abscissa of a highest wave crest (subharmonic bifurcations yield crests of different heights) or of a deepest trough for aperiodic waves of depression when there is only one axis of symmetry.

$y=-d, y=\eta(x)$ and $y=0$ denote, respectively, the equations for the bottom, for the free surface and for the mean water level. The latter implies that $\langle\eta\rangle=0-\langle\bullet\rangle$ the Eulerian average operator over one period - i.e.

$$
\langle\eta\rangle \stackrel{\text { def }}{=} \frac{k}{2 \pi} \int_{-\pi / k}^{\pi / k} \eta(x) \mathrm{d} x=0 .
$$


$a \stackrel{\text { def }}{=} \eta(0)$ denotes the wave amplitude (i.e., the mean level to the crest elevation) and $b \stackrel{\text { def }}{=}-\eta(\pi / k)$ is the trough elevation; hence $a+b$ is the total wave height if the surface varies monotonically from crest to trough. A wave steepness $\varepsilon$ is then classically defined as $\varepsilon \stackrel{\text { def }}{=} k(a+b) / 2$.

Let be $\phi, \psi, u$ and $v$ the velocity potential, the stream function, the horizontal and vertical velocities, respectively, such that $u=\phi_{x}=\psi_{y}$ and $v=\phi_{y}=-\psi_{x}$. It is convenient to introduce the complex potential $f \stackrel{\text { def }}{=} \phi+\mathrm{i} \psi\left(\right.$ with $\left.\mathrm{i}^{2}=-1\right)$ and the complex velocity $w \stackrel{\text { def }}{=} u-\mathrm{i} v$; these are holomorphic functions of $z \stackrel{\text { def }}{=} x+\mathrm{i} y$ (i.e., $f=f(z)$ and $w(z)=\mathrm{d} f / \mathrm{d} z$ ). The complex conjugate is denoted with a star (e.g., $\left.z^{*}=x-\mathrm{i} y\right)$, while over-tildes denote the quantities written at the surface - e.g., $\tilde{z}(x)=x+\mathrm{i} \eta(x), \tilde{\phi}(x)=\phi(x, y=\eta(x))$. (Note that, e.g., $\tilde{u}=\widetilde{\phi_{x}} \neq \tilde{\phi}_{x}=\tilde{u}+\eta_{x} \tilde{v}$.) Because the free surface and bottom are streamlines, $\tilde{\psi}=\psi(x, \eta)$ and $\psi(x,-d)$ are constants.

The dynamic condition can be expressed in term of the Bernoulli equation,

$$
2 p+2 g y+u^{2}+v^{2}=B,
$$

where $g>0$ is the acceleration due to gravity and $B$ is a Bernoulli constant. At the free surface the pressure reduces to the effect of the surface tension, i.e., $\tilde{p}=-\tau \eta_{x x}\left(1+\eta_{x}^{2}\right)^{-3 / 2}$, $\tau$ being a surface tension coefficient divided by the density. Let $-c$ be the mean velocity at the bed such that

$$
c \stackrel{\text { def }}{=}-\langle u(x, y=-d)\rangle .
$$

Thus, $c$ is the phase velocity of the wave observed in the frame of reference where the mean velocity at the bottom is zero, and $c>0$ if the wave travels in the increasing $x$-direction. A definition of $c$ is not needed for solving the equations in the frame of reference moving with the wave, where the flow is steady. A definition of $c$ is however necessary when the solution needs to be eventually expressed in another frame of reference. The latter can be obtained via a Galilean transformation properly done [10].

Note that many different phase velocities can be defined (i.e., many frames of reference can be of practical interest), and defining phase velocities is not a trivial matter, in general [39]. Here, $c$ is Stokes' first definition of wave celerity [10, 36] and comparisons with another phase velocity can be found in [16]. Note also that this frame issue also appears when investigating, for example, fluid particle trajectories [14, 31, 41] since the latter are different in different frames (i.e., trajectories are not Galilean invariant). In the frame of reference moving with the wave, trajectories coincide with streamlines (and streaklines) because the flow is steady in this peculiar frame. Trajectories in any other frames can then be obtained via Galilean transformations. Note finally that $B=c^{2}$ in deep water and for solitary waves (but $B \neq c^{2}$ in general) and that $c$ is not the linear phase velocity $c_{0} \stackrel{\text { def }}{=} \sqrt{\left(g+\tau k^{2}\right) \tanh (k d) / k}$.

All the equations in this paper are for non-overturning waves, so $\eta$ is a continuous function of $x$. For pure gravity waves, there are no overhanging travelling waves of permanent form [34, 43]. The situation is different in presence of surface tensions where overhanging solutions exist [27, 42]. Because we derive below some equations for $\eta$ only, their 
generalisation for overturning waves is straightforward, using for instance the arc length coordinate as independent variable. Thus, using $x$ as independent does not really restrict the generality of the derivations below.

\section{Surface Dynamic Relations}

A steady impermeable free surface implying that $\tilde{\psi}$ is a constant, the Bernoulli equation (2) at the free surface can be written

$$
2 g \eta+\tilde{\phi}_{x}^{2}\left(1+\eta_{x}^{2}\right)^{-1}-2 \tau \eta_{x x}\left(1+\eta_{x}^{2}\right)^{-3 / 2}=B .
$$

Hence, the Bernoulli constant $B$ is such that

$$
B=\left\langle\tilde{\phi}_{x}^{2}\left(1+\eta_{x}^{2}\right)^{-1}\right\rangle=\left\langle\phi_{x}^{2}(x,-d)\right\rangle,
$$

the first equality being a consequence of the condition (1) and of the periodicity applied to (44), while the second equality derives from the irrotationality [11, 24].

The relation $w=\mathrm{d} f / \mathrm{d} z$ written at the free surface, with the equation (4), yields [11]

$$
\begin{aligned}
\tilde{w}^{2} & =\left(\frac{\mathrm{d} \tilde{f}}{\mathrm{~d} \tilde{z}}\right)^{2}=\left(\frac{\mathrm{d} \tilde{f}}{\mathrm{~d} x} / \frac{\mathrm{d} \tilde{z}}{\mathrm{~d} x}\right)^{2}=\left(\frac{\tilde{\phi}_{x}}{1+\mathrm{i} \eta_{x}}\right)^{2}=\frac{\tilde{\phi}_{x}^{2}}{1+\eta_{x}^{2}} \frac{1-\mathrm{i} \eta_{x}}{1+\mathrm{i} \eta_{x}} \\
& =\left[B-2 g \eta+2 \tau \eta_{x x}\left(1+\eta_{x}^{2}\right)^{-3 / 2}\right]\left(1-\mathrm{i} \eta_{x}\right) /\left(1+\mathrm{i} \eta_{x}\right) .
\end{aligned}
$$

$w^{2}$ being a holomorphic function, the function 9

$$
\mathfrak{Q}(z) \stackrel{\text { def }}{=} \int_{z_{0}}^{z} \frac{1}{2}\left[B-w^{2}\left(z^{\prime}\right)\right] \mathrm{d} z^{\prime},
$$

$\left(z_{0}\right.$ an arbitrary constant) is of course also holomorphic. Taking $z_{0}$ at the wave crest (i.e., choosing $z_{0}=\mathrm{i} a$ ), integrating along the surface and using (6), one obtains at once

$$
\begin{aligned}
\widetilde{\mathfrak{Q}}(x) & =\int_{0}^{x} \frac{1}{2}\left[B-w^{2}\left(z^{\prime}\right)\right]\left[1+\mathrm{i} \eta_{x}\left(x^{\prime}\right)\right] \mathrm{d} x^{\prime} \\
& =g H-\frac{\mathrm{i}}{2} g(\eta-a)(\eta+a-2 B / g)+\mathrm{i} \tau-\tau\left(\mathrm{i}+\eta_{x}\right) / \sqrt{1+\eta_{x}^{2}},
\end{aligned}
$$

where $H(x) \stackrel{\text { def }}{=} \int_{0}^{x} \eta\left(x^{\prime}\right) \mathrm{d} x^{\prime}$. For symmetric waves, $H$ is a periodic odd function and therefore averages to zero because $\eta$ is a periodic even function averaging to zero. For asymmetric waves, $H$ is still periodic but, a priori, does not necessarily have zero average.

It follows from (5) and from the bottom impermeability that on the seabed

$$
\left\langle\operatorname{Re}\left\{\mathfrak{Q}_{z}\right\}\right\rangle=0 \quad \text { and } \operatorname{Im}\left\{\mathfrak{Q}_{z}\right\}=0 \quad \text { at } z=x-\mathrm{i} d .
$$

Thus, $\mathfrak{Q}$ is a $(2 \pi / k)$-periodic function in the $x$-direction that is bounded everywhere at and below the free surface, even in the deep water limit $d \rightarrow \infty$.

The mathematical formulation involving the function $\mathfrak{Q}$ has the great advantage that the conditions at the free surface are combined into a single complex equation. Thus, the holomorphic properties can be exploited in a straightforward efficient way, as shown in [9]. 


\section{Fourier expansion And Poisson-Like integral}

For a $(2 \pi / k)$-periodic wave, such that the boundary conditions (9) are fulfilled, a general solution can be sought as the Fourier expansion

$$
\mathfrak{Q}=\mathrm{i} \mathfrak{q}_{0}+\mathrm{i} \sum_{n=1}^{\infty}\left[\mathfrak{q}_{n} \mathrm{e}^{-\mathrm{i} n k z}-\mathfrak{q}_{n}^{*} \mathrm{e}^{\mathrm{i} n k(z+2 \mathrm{i} d)}\right],
$$

where all the Fourier coefficients $\mathfrak{q}_{n}$ are real if the wave is symmetric with respect to the vertical axis $x=0$. The condition $\widetilde{\mathfrak{Q}}(0)=0$ (from the definition of $\mathfrak{Q}$ ) yields

$$
\mathfrak{q}_{0}=\sum_{n=1}^{\infty}\left[\mathfrak{q}_{n}^{*} \mathrm{e}^{-2 n k(a+d)}-\mathfrak{q}_{n}\right] \mathrm{e}^{n k a} .
$$

For later convenience, we introduce another holomorphic function $q$ :

$$
q(z) \stackrel{\text { def }}{=} \mathfrak{Q}(z)-\mathrm{i} \mathfrak{q}_{0}=\mathrm{i} \sum_{n=1}^{\infty}\left[\mathfrak{q}_{n} \mathrm{e}^{-\mathrm{i} n k z}-\mathfrak{q}_{n}^{*} \mathrm{e}^{\mathrm{i} n k(z+2 \mathrm{i} d)}\right],
$$

such that at the bottom $\langle q(x-\mathrm{i} d)\rangle=0, \operatorname{Im}\{q(x-\mathrm{i} d)\}=0$ and at the free surface $\left\langle\tilde{q} \tilde{z}_{x}\right\rangle=0$.

The coefficients $\mathfrak{q}_{n}$ can be obtained from $\tilde{\mathfrak{Q}}(x)=\mathfrak{Q}(\tilde{z})$ via the relations (spectral projections)

$$
\left\langle\mathfrak{Q}(\tilde{z}) \exp (\mathrm{i} n k \tilde{z}) \frac{\mathrm{d} \tilde{z}}{\mathrm{~d} x}\right\rangle= \begin{cases}\mathrm{i} \mathfrak{q}_{n} \operatorname{if} & n \geqslant 0 \\ -\mathrm{i} \mathfrak{q}_{-n}^{*} \exp (2 n k d) & \text { if } \quad n<0\end{cases}
$$

the equality deriving from the obvious relation

$$
\left\langle\exp (\operatorname{ink} \tilde{z}) \frac{\mathrm{d} \tilde{z}}{\mathrm{~d} x}\right\rangle= \begin{cases}1 & (n=0), \\ 0 & (n \neq 0) .\end{cases}
$$

Integrating by parts and using the condition (11), one derives easily the similar relations

$$
\begin{aligned}
\left\langle\eta \exp (\mathrm{i} n k \tilde{z}) \frac{\mathrm{d} \tilde{z}}{\mathrm{~d} x}\right\rangle & = \begin{cases}0 & (n=0), \\
-\langle\exp (\mathrm{i} n k \tilde{z})\rangle /(n k) & (n \neq 0),\end{cases} \\
\left\langle\eta^{2} \exp (\mathrm{i} n k \tilde{z}) \frac{\mathrm{d} \tilde{z}}{\mathrm{~d} x}\right\rangle & = \begin{cases}\left\langle\eta^{2}\right\rangle & (n=0), \\
-2\langle(1+n k \eta) \exp (\mathrm{i} n k \tilde{z})\rangle /(n k)^{2} & (n \neq 0),\end{cases} \\
\left\langle H \exp (\mathrm{i} n k \tilde{z}) \frac{\mathrm{d} \tilde{z}}{\mathrm{~d} x}\right\rangle & = \begin{cases}\langle H\rangle-\mathrm{i}\left\langle\eta^{2}\right\rangle & (n=0), \\
\mathrm{i}\langle\eta \exp (\mathrm{i} n k \tilde{z})\rangle /(n k) & (n \neq 0),\end{cases}
\end{aligned}
$$

as well as (for all $n \in \mathbb{Z}$ )

$$
\left\langle\frac{\mathrm{i}+\eta_{x}}{\sqrt{1+\eta_{x}^{2}}} \exp (\mathrm{i} n k \tilde{z}) \frac{\mathrm{d} \tilde{z}}{\mathrm{~d} x}\right\rangle=\mathrm{i}\left\langle\sqrt{1+\eta_{x}^{2}} \exp (\mathrm{i} n k \tilde{z})\right\rangle,
$$

where $\langle H\rangle \neq 0$ only (perhaps) for asymmetric waves. Note that the right-hand sides of (15), (16) and (17) do not involve the derivatives of $\eta$, which is an interesting feature for 
computations. With the relations (15)-(18), the substitution of (8) into (13) yields

$$
\begin{aligned}
\mathfrak{q}_{0} & =\frac{1}{2} g a^{2}-\frac{3}{2} g\left\langle\eta^{2}\right\rangle-B a-\mathrm{i} g\langle H\rangle+\tau-\tau\left\langle\sqrt{1+\eta_{x}^{2}}\right\rangle & & (n=0), \\
\mathfrak{q}_{n} & =\left\langle\left[\frac{g}{(n k)^{2}}-\frac{B}{n k}+\frac{2 g \eta}{n k}-\tau \sqrt{1+\eta_{x}^{2}}\right] \mathrm{e}^{\mathrm{i} n k \tilde{z}}\right\rangle & & (n>0), \\
-\mathfrak{q}_{-n}^{*} \mathrm{e}^{2 n k d} & =\left\langle\left[\frac{g}{(n k)^{2}}-\frac{B}{n k}+\frac{2 g \eta}{n k}-\tau \sqrt{1+\eta_{x}^{2}}\right] \mathrm{e}^{\mathrm{i} n k \tilde{z}}\right\rangle & & (n<0),
\end{aligned}
$$

which gives all the $\mathfrak{q}_{n}$ if $\eta$ is known. Hence $\mathfrak{Q}$ and $q$ are completely determined in terms of $\eta$, allowing the derivation of equations for $\eta$ only, as shown in the sections 5 and 6 below. Note that the $\mathfrak{q}_{n}$ (for $n \neq 0$ ) and $q$ at the free surface, i.e. from (8) and (19)

$$
\tilde{q}=g(H-\langle H\rangle)-\frac{\mathrm{i}}{2} g\left(\eta^{2}-3\left\langle\eta^{2}\right\rangle\right)+\mathrm{i} B \eta-\tau\left(\mathrm{i}+\eta_{x}\right)\left(1+\eta_{x}^{2}\right)^{-1 / 2}+\mathrm{i} \tau\left\langle\sqrt{1+\eta_{x}^{2}}\right\rangle,
$$

do not depend explicitly on the amplitude $a$.

Once $\eta$ is known, the function $q$ is obtained from its Fourier expansion (12). Substituting the relation (20), one derives the Poisson-like integral relation

$$
\begin{aligned}
q(z) & =\mathrm{i} \sum_{n=1}^{\infty}\left[\mathfrak{q}_{n} \mathrm{e}^{-\mathrm{i} n k z}-\mathfrak{q}_{n}^{*} \mathrm{e}^{\mathrm{i} n k(z+2 \mathrm{i} d)}\right] \\
& =\frac{\mathrm{i} k}{2 \pi} \int_{-\frac{\pi}{k}}^{\frac{\pi}{k}} \sum_{n=1}^{\infty}\left[\frac{g}{(n k)^{2}}-\frac{B-2 g \eta^{\prime}}{n k}+\tau \sqrt{1+\eta_{x}^{\prime 2}}\right]\left[\mathrm{e}^{\mathrm{i} n k\left(\tilde{z}^{\prime}-z\right)}-\mathrm{e}^{-\mathrm{i} n k\left(\tilde{z}^{* \prime}-z-2 \mathrm{i} d\right)}\right] \mathrm{d} x^{\prime} \\
& =\frac{\mathrm{i} k}{2 \pi} \int_{-\frac{\pi}{k}}^{\frac{\pi}{k}}\left[\frac{g}{k^{2}} \mathscr{L}_{2}-\frac{B-2 g \eta^{\prime}}{k} \mathscr{L}_{1}+\tau \sqrt{1+\eta_{x}^{\prime 2}} \mathscr{L}_{0}\right] \mathrm{d} x^{\prime},
\end{aligned}
$$

with the kernels (for $\nu=0,1,2$ )

$$
\mathscr{L}_{\nu} \stackrel{\text { def }}{=} \operatorname{Li}_{\nu}\left(\mathrm{e}^{\mathrm{i} k\left(\tilde{z}^{\prime}-z\right)}\right)-\left[\operatorname{Li}_{\nu}\left(\mathrm{e}^{\mathrm{i} k\left(\tilde{z}^{\prime}-z^{*}+2 \mathrm{i} d\right)}\right)\right]^{*}=\operatorname{Li}_{\nu}\left(\mathrm{e}^{\mathrm{i} k\left(\tilde{z}^{\prime}-z\right)}\right)-\operatorname{Li}_{\nu}\left(\mathrm{e}^{\mathrm{i} k\left(z-\tilde{z}^{* \prime}+2 \mathrm{i} d\right)}\right)
$$

the primes denoting the dependence with respect to the dummy variable $x^{\prime}$ (e.g., $\eta^{\prime}=\eta\left(x^{\prime}\right)$, $\tilde{z}^{\prime}=x^{\prime}+\mathrm{i} \eta\left(x^{\prime}\right)$, etc.) and where $\operatorname{Li}_{\nu}(\theta) \stackrel{\text { def }}{=} \sum_{n=1}^{\infty} \theta^{n} / n^{\nu}$ is the $\nu$-th polylogarithm [20]. In particular, $\operatorname{Li}_{0}(\theta) \stackrel{\text { def }}{=} \theta /(1-\theta), \operatorname{Li}_{1}(\theta) \stackrel{\text { def }}{=}-\log (1-\theta)$ and $\operatorname{Li}_{2}(\theta)$ cannot be expressed with simpler function 1$]$ but it can be easily computed [6]. $\operatorname{Li}_{\nu}(\theta)$ for $\nu \geqslant 1$ is a single-valued function in the cut plane $\theta \in \mathbb{C} \backslash\left[1, \infty\left[\right.\right.$, i.e., with $\theta=\mathrm{e}^{\mathrm{i} k\left(\tilde{z}^{\prime}-z\right)}$ the branch cut is defined by $x=x^{\prime}$ and $y \geqslant \eta^{\prime}$. Therefore, the relation (23) is well defined for all $z$ beneath the free surface.

Once $q$ is known, the velocity field is obtained from the relation $w^{2}=B-2 \mathrm{~d} q / \mathrm{d} z$. The pressure and other (e.g. acceleration) fields can then be obtained from $w$ via some elementary mathematical derivations.

\footnotetext{
${ }^{1} \mathrm{Li}_{2}$ is Spence's dilogarithm, which is not to be confused with Euler's dilogarithm $\operatorname{dlog}(\theta) \stackrel{\text { def }}{=} \mathrm{Li}_{2}(1-\theta)$.

${ }^{2}$ Only the principal branch (such that $-\pi<\arg (\theta) \leqslant \pi$ ) of the complex logarithm is considered here. This convention defines the branch cut of $\mathrm{Li}_{1}$, which is carried out to the definition of higher-order complex polylogarithms via the recurrence relation $\operatorname{Li}_{\nu+1}(\theta)=\int_{0}^{\theta} \theta^{\prime-1} \operatorname{Li}_{\nu}\left(\theta^{\prime}\right) \mathrm{d} \theta^{\prime}$.
} 
5. System OF EQUATIONS FOR the Free SURFACE

Changing $n$ by $-n$ in the complex conjugate of (21) and subsequently substituting (20) into the result in order to eliminate $\mathfrak{q}_{n}$, one obtains after some elementary algebra (for all $n>0)$

$$
\left\langle\left[\frac{g}{(n k)^{2}}+\frac{B-2 g \eta}{n k}-\tau \sqrt{1+\eta_{x}^{2}}\right] \mathrm{e}^{\mathrm{i} n k \tilde{z}^{*}}+\left[\frac{g}{(n k)^{2}}-\frac{B-2 g \eta}{n k}-\tau \sqrt{1+\eta_{x}^{2}}\right] \mathrm{e}^{\mathrm{i} n k(\tilde{z}+2 \mathrm{i} d)}\right\rangle=0 .
$$

The (infinite) system of equations (25), together with the condition (1), determines completely the surface elevation $\eta$. Therefore, this system of equations can be used to compute $\eta$. These relations can be also used to check the accuracy of numerical solutions obtained from any formulation of the problem, thus providing a much more stringent criterion of convergence and accuracy than the few well-known integral relations [24, 26, 35] often used for this purpose.

For symmetric gravity waves in deep water - i.e., when $\eta(-x)=\eta(x), \tau=0, d=\infty$ and $B=c^{2}$ - the equation (25) is significantly simplified as

$$
\left\langle\left[g(n k)^{-1}+c^{2}-2 g \eta\right] \exp (n k \eta) \cos (n k x)\right\rangle=0 \quad n=1,2,3, \cdots .
$$

This simple relation is suitable, in particular, for computing the coefficients $\mathfrak{a}_{m, j}$ of the Stokes double expansion

$$
\eta=\sum_{m=1}^{\infty} \sum_{j=0}^{\infty} \epsilon^{m+j} \mathfrak{a}_{m, j} \cos (m k x)
$$

where $\epsilon$ is a small parameter. With (26) , this calculation is not much more demanding in the physical plane than in the conformal one, and it has the advantage that the Stokes expansion has a better rate of convergence [7].

It should be noted that (26) is somehow a physical plane counterpart of the quadratic relations between the Fourier coefficients of the Stokes waves obtained by Longuet-Higgins [25] in the conformal plane. The relation (25) is the generalisation for finite depth and surface tensions, whose conformal plane counterpart has apparently never been derived.

\section{INTEGRAL EQUATIONS FOR THE FREE SURFACE}

Applying (23) at the free surface and substituting (22) in the left-hand side, one obtains

$$
\begin{aligned}
g(H-\langle H\rangle) & -\frac{\mathrm{i}}{2} g\left(\eta^{2}-3\left\langle\eta^{2}\right\rangle\right)+\mathrm{i} B \eta-\tau\left(\mathrm{i}+\eta_{x}\right)\left(1+\eta_{x}^{2}\right)^{-1 / 2}+\mathrm{i} \tau\left\langle\sqrt{1+\eta_{x}^{2}}\right\rangle \\
& =\frac{\mathrm{i} k}{2 \pi} f_{-\frac{\pi}{k}}^{\frac{\pi}{k}}\left[\frac{g}{k^{2}} \widetilde{\mathscr{L}_{2}}-\frac{B-2 g \eta^{\prime}}{k} \widetilde{\mathscr{L}}_{1}+\tau \sqrt{1+\eta_{x}^{\prime 2}} \widetilde{\mathscr{L}}_{0}\right] \mathrm{d} x^{\prime},
\end{aligned}
$$

$f$ denoting a singular integral to be evaluated in the sense of Cauchy principal value and where

$$
\widetilde{\mathscr{L}_{\nu}} \stackrel{\text { def }}{=} \operatorname{Li}_{\nu}\left(\mathrm{e}^{\mathrm{i} k\left(\tilde{z}^{\prime}-\tilde{z}\right)}\right)-\left[\operatorname{Li}_{\nu}\left(\mathrm{e}^{\mathrm{i} k\left(\tilde{z}^{\prime}-\tilde{z}^{*}+2 \mathrm{i} d\right)}\right)\right]^{*},
$$

the kernel $\widetilde{\mathscr{L}}_{0}$ being singular (hence the principal value integral), $\widetilde{\mathscr{L}}_{1}$ being weakly singular (with logarithmic singularity) and $\widetilde{\mathscr{L}}_{2}$ being regular. Thus, splitting the real and imaginary 
parts of (28) $)$, i.e.,

$$
\begin{gathered}
g H-g\langle H\rangle-\tau \eta_{x}\left(1+\eta_{x}^{2}\right)^{-1 / 2} \\
=-\frac{k}{2 \pi} \int_{-\frac{\pi}{k}}^{\frac{\pi}{k}}\left[\frac{g}{k^{2}} \operatorname{Im}\left\{\widetilde{\mathscr{L}}_{2}\right\}-\frac{B-2 g \eta^{\prime}}{k} \operatorname{Im}\left\{\widetilde{\mathscr{L}}_{1}\right\}+\tau \sqrt{1+\eta_{x}^{\prime 2}} \operatorname{Im}\left\{\widetilde{\mathscr{L}_{0}}\right\}\right] \mathrm{d} x^{\prime}, \\
B \eta-\frac{1}{2} g \eta^{2}+\frac{3}{2} g\left\langle\eta^{2}\right\rangle-\tau\left(1+\eta_{x}^{2}\right)^{-1 / 2}+\tau\left\langle\sqrt{1+\eta_{x}^{2}}\right\rangle \\
=\frac{k}{2 \pi} f_{-\frac{\pi}{k}}^{\frac{\pi}{k}}\left[\frac{g}{k^{2}} \operatorname{Re}\left\{\widetilde{\mathscr{L}}_{2}\right\}-\frac{B-2 g \eta^{\prime}}{k} \operatorname{Re}\left\{\widetilde{\mathscr{L}}_{1}\right\}+\tau \sqrt{1+\eta_{x}^{\prime 2}} \operatorname{Re}\left\{\widetilde{\mathscr{L}}_{0}\right\}\right] \mathrm{d} x^{\prime},
\end{gathered}
$$

one obtains two conjugate nonlinear singular integro-differential equations for $\eta$. Either equation (30) or equation (31) can be used to compute the solution, the other one can be used to check the accuracy of the computed approximation.

For pure gravity waves $(\tau=0)$, the imaginary part (31) of (28) becomes

$$
\begin{aligned}
2 B \eta- & g \eta^{2}+3 g\left\langle\eta^{2}\right\rangle=\frac{g}{\pi k} \int_{-\frac{\pi}{k}}^{\frac{\pi}{k}} \operatorname{Re}\left\{\operatorname{Li}_{2}\left(\mathrm{e}^{\mathrm{i} k\left(\tilde{z}^{\prime}-\tilde{z}\right)}\right)-\operatorname{Li}_{2}\left(\mathrm{e}^{\mathrm{i} k\left(\tilde{z}-\tilde{z}^{* \prime}+2 \mathrm{i} d\right)}\right)\right\} \mathrm{d} x^{\prime} \\
- & \frac{1}{\pi} \int_{-\frac{\pi}{k}}^{\frac{\pi}{k}}\left(B-2 g \eta^{\prime}\right) \operatorname{Re}\left\{\operatorname{Li}_{1}\left(\mathrm{e}^{\mathrm{i} k\left(\tilde{z}^{\prime}-\tilde{z}\right)}\right)-\operatorname{Li}_{1}\left(\mathrm{e}^{\mathrm{i} k\left(\tilde{z}-\tilde{z}^{* \prime}+2 \mathrm{i} d\right)}\right)\right\} \mathrm{d} x^{\prime}
\end{aligned}
$$

which is a nonlinear weakly singular purely integral (i.e., not differential) equation for $\eta$. Equation (32) is the physical plane counterpart of the Babenko equation [1, the latter being expressed in the conformal plane. Thus, we name equation (32) the "Eulerian Babenko equation" (EBE) and the original Babenko equation is called here the "conformal Babenko equation" (CBE). The CBE is most often written with pseudo-differential operators that can also be expressed as convolution integrals. In doing so, one can easily verify that the CBE involves hyper-singular integrals, while the EBE involves only weakly-singular integrals. However, the integrals of the EBE are not of convolution type and their kernels involve the unknown function $\eta$. Except for the highest waves, the CBE can be easily and rapidly solved numerically [12]. However, the CBE is not so convenient for extreme waves for which the EBE is expected to be superior.

The Nekrasov equation [29] is sometimes used to compute extreme gravity waves [3, 28]. Like the EBE, Nekrasov's integral equation is only weakly singular, but this equation is for the angle the free surface makes with the horizontal. This angle being discontinuous at the crest of the limiting waves, the numerical resolution of the Nekrasov equation is demanding for near-limiting waves. Conversely, the EBE being an equation for the surface itself that is a continuous function, even for the limiting gravity waves, it has an attractive feature for extreme waves.

Equation (31) is an EBE generalised to incorporate surface tensions. The CBE with surface tension has long been derived and it was used in mathematical and numerical analysis [2, 13]. Equation (31) involves first-order derivatives while the CBE with surface tension involves second-order derivatives. Therefore, equation (31) should be useful for further mathematical and numerical investigations. 


\section{REgularised integral EQUATION}

When capillarity is included, the singular nature of the EBE is not really problematic because the surface tension enforces some regularity of the free surface. However, for steep pure gravity waves, such as the limiting waves with a $120^{\circ}$ inner angle at the crest, the singular integral is more problematic due to the low regularity of $\eta$, even though the integral is only weakly singular. It is therefore of practical interest to regularise the equation.

The integral equation (31) has a (weak, logarithmic) singularity due to the term $\operatorname{Li}_{1}\left(\mathrm{e}^{\mathrm{i} k\left(\tilde{z}^{\prime}-\tilde{z}\right)}\right)$ and has a (single pole) singularity due to the term $\operatorname{Li}_{0}\left(\mathrm{e}^{\mathrm{i} k\left(\tilde{z}^{\prime}-\tilde{z}\right)}\right)$. Both singularities can be removed as follows. First, the equation (31) is rewritten as

$$
\begin{gathered}
2 B \eta-g \eta^{2}+3 g\left\langle\eta^{2}\right\rangle-2 \tau\left(1+\eta_{x}^{2}\right)^{-1 / 2}+2 \tau\left\langle\sqrt{1+\eta_{x}^{2}}\right\rangle=\frac{g}{\pi k} \int_{-\frac{\pi}{k}}^{\frac{\pi}{k}} \operatorname{Re}\left\{\widetilde{\mathscr{L}}_{2}\right\} \mathrm{d} x^{\prime} \\
-\frac{2 g}{\pi} \int_{-\frac{\pi}{k}}^{\frac{\pi}{k}}\left(\eta-\eta^{\prime}\right) \operatorname{Re}\left\{\widetilde{\mathscr{L}}_{1}\right\} \mathrm{d} x^{\prime}-\frac{B-2 g \eta}{\pi} \int_{-\frac{\pi}{k}}^{\frac{\pi}{k}} \operatorname{Re}\left\{\widetilde{\mathscr{L}}_{1}\right\} \mathrm{d} x^{\prime} \\
+\frac{k \tau}{\pi} \int_{-\frac{\pi}{k}}^{\frac{\pi}{k}}\left(\sqrt{1+\eta_{x}^{\prime 2}}-\sqrt{1+\eta_{x}^{2}}\right) \operatorname{Re}\left\{\widetilde{\mathscr{L}_{0}}\right\} \mathrm{d} x^{\prime}+\frac{k \tau}{\pi} \sqrt{1+\eta_{x}^{2}} f_{-\frac{\pi}{k}}^{\frac{\pi}{k}} \operatorname{Re}\left\{\widetilde{\mathscr{L}}_{0}\right\} \mathrm{d} x^{\prime},
\end{gathered}
$$

where only the third and fifth integrands are singular. Second, considering the relations

$$
\overline{\mathscr{L}_{\nu}} \stackrel{\text { def }}{=} \operatorname{Li}_{\nu}\left(\mathrm{e}^{\mathrm{i} k\left(x^{\prime}-x\right)}\right)-\operatorname{Li}_{\nu}\left(\mathrm{e}^{\mathrm{i} k\left(x-x^{\prime}+2 \mathrm{i} d\right)}\right), \quad \int_{-\frac{\pi}{k}}^{\frac{\pi}{k}} \operatorname{Li}_{\nu}\left(\mathrm{e}^{\mathrm{i} k\left(x^{\prime}-x+\mathrm{i} \alpha\right)}\right) \mathrm{d} x^{\prime}=0,
$$

where $\alpha \in \mathbb{R}^{+}$is a constant, the equation (33) can be further rewritten

$$
\begin{gathered}
2 B \eta-g \eta^{2}+3 g\left\langle\eta^{2}\right\rangle-2 \tau\left(1+\eta_{x}^{2}\right)^{-1 / 2}+2 \tau\left\langle\sqrt{1+\eta_{x}^{2}}\right\rangle= \\
-\frac{2 g}{\pi} \int_{-\frac{\pi}{k}}^{\frac{\pi}{k}}\left(\eta-\eta^{\prime}\right) \operatorname{Re}\left\{\widetilde{\mathscr{L}}_{1}\right\} \mathrm{d} x^{\prime}+\frac{k \tau}{\pi} \int_{-\frac{\pi}{k}}^{\frac{\pi}{k}}\left(\sqrt{1+\eta_{x}^{\prime 2}}-\sqrt{1+\eta_{x}^{2}}\right) \operatorname{Re}\left\{\widetilde{\mathscr{L}_{0}}\right\} \mathrm{d} x^{\prime} \\
+\frac{g}{\pi k} \int_{-\frac{\pi}{k}}^{\frac{\pi}{k}} \operatorname{Re}\left\{\widetilde{\mathscr{L}_{2}}-\overline{\mathscr{L}_{2}}\right\} \mathrm{d} x^{\prime}-\frac{B-2 g \eta}{\pi} \int_{-\frac{\pi}{k}}^{\frac{\pi}{k}} \operatorname{Re}\left\{\widetilde{\mathscr{L}_{1}}-\overline{\mathscr{L}_{1}}\right\} \mathrm{d} x^{\prime} \\
+\frac{k \tau}{\pi} \sqrt{1+\eta_{x}^{2}} \int_{-\frac{\pi}{k}}^{\frac{\pi}{k}} \operatorname{Re}\left\{\widetilde{\mathscr{L}}_{0}-\left(1+\mathrm{i} \eta_{x}\right)^{-1} \overline{\mathscr{L}_{0}}\right\} \mathrm{d} x^{\prime} .
\end{gathered}
$$


All the integrands in (35) are continuous if $\eta$ is continuous (non-overturning waves) because as $x^{\prime} \rightarrow x$ we have

$$
\begin{aligned}
\operatorname{Re}\left\{\widetilde{\mathscr{L}}_{0}-\left(1+\mathrm{i} \eta_{x}\right)^{-1} \overline{\mathscr{L}_{0}}\right\} & \rightarrow O\left(\left(x^{\prime}-x\right)^{0}\right), \\
\left(\sqrt{1+\eta_{x}^{\prime 2}}-\sqrt{1+\eta_{x}^{2}}\right) \operatorname{Re}\left\{\widetilde{\mathscr{L}}_{0}\right\} & \rightarrow \frac{\eta_{x x} \eta_{x}^{2}}{k\left(1+\eta_{x}^{2}\right)^{3 / 2}}, \\
\operatorname{Re}\left\{\widetilde{\mathscr{L}_{1}}-\overline{\mathscr{L}_{1}}\right\} & \rightarrow \log \left(\frac{1-\mathrm{e}^{-2 k(\eta+d)}}{\left(1-\mathrm{e}^{-2 k d}\right) \sqrt{1+\eta_{x}^{2}}}\right), \\
\left(\eta-\eta^{\prime}\right) \operatorname{Re}\left\{\widetilde{\mathscr{L}}_{1}\right\} & \rightarrow 0, \\
\operatorname{Re}\left\{\widetilde{\mathscr{L}}_{2}-\overline{\mathscr{L}_{2}}\right\} & \rightarrow \operatorname{Li}_{2}\left(\mathrm{e}^{-2 k d}\right)-\operatorname{Li}_{2}\left(\mathrm{e}^{-2 k(\eta+d)}\right),
\end{aligned}
$$

the $O\left(\left(x^{\prime}-x\right)^{0}\right)$ term on the right-hand side of (36) being too complicated to be reported here. The additional dilogarithmic term is not necessary because $\widetilde{\mathscr{L}}_{2}$ is regular as $x^{\prime} \rightarrow x$. The $\overline{\mathscr{L}_{2}}$ term has nevertheless been subtracted in order to improve the equation in view of numerical computations.

When $\tau=0$ (pure gravity waves), (35) is a regular integral equation for $\eta$. The absence of derivatives (of the unknown dependent variable $\eta$ ) in this regular integral equation is $a$ priori an interesting feature for computing extreme waves.

\section{Conclusion}

Several exact relations for steady irrotational surface waves have been derived. The derivations were carried out in the physical plane and it was shown that these relations are not much more involved than their counterparts in the conformal plane. In particular, we derived several integral relations for the free surface only that can be of practical interest. We also obtained integral equations for the free surface that can be used to investigate analytically and numerically steady water waves. The main motivation for this work was the derivation of equations suitable to study extreme waves, but the practical benefit of these equations remains to be demonstrated. Nonetheless, we emphasised several of their features that seem advantageous a priori. It will be the subject of future investigations to explore the potential of these equations, for instance exploiting equation (32) to compute accurately the highest waves.

The integral relations and equations derived in this paper are valid for non-overturning waves, i.e., $\eta$ must be a single valued continuous function of $x$. For gravity-capillary waves, some overturning solutions are known to exist [27, 42]. For such solutions, using $x$ as independent variable is no longer possible. One should then use instead a parametric representation of the free surface, such as the arc length coordinate. The corresponding integral relations and equations can be easily obtained from (25), (30) and (31). These elementary derivations are left to the reader.

This work could be extended in different directions. Generalisations for overturning waves are straightforward using, for instance, the arc length coordinate as independent variable, as already mentioned. The inclusion of elastic effects at the surface modelling 
flexural surface waves [40] should also be straightforward. Stratifications in homogeneous layers could be treated in similar ways. The inclusion of vorticity would be of special interest and it seems conceivable, at least for a constant vorticity. These possibilities will be investigated in future works.

\section{REFERENCES}

[1] K. I. Babenko. Some remarks on the theory of surface waves of finite amplitude. Sov. Math. Dokl., 35:599-603, 1987.

[2] B. Buffoni, E. N. Dancer, and J. F. Toland. The regularity and local bifurcation of steady periodic water waves. Arch. Rat. Mech. Anal., 152(3):207-240, jun 2000.

[3] J. G. B. Byatt-Smith. Numerical solution of Nekrasov's equation in the boundary layer near the crest for waves near the maximum height. Stud. App. Math., 106:393-405, 2001.

[4] D. Clamond. Steady finite-amplitude waves on a horizontal seabed of arbitrary depth. J. Fluid Mech., 398:45-60, 1999.

[5] D. Clamond. Cnoidal-type surface waves in deep water. J. Fluid Mech., 489:101-120, 2003.

[6] D. Clamond. Complex Dilogarithm. Matlab File Exchange, 2006.

[7] D. Clamond. On the Lagrangian description of steady surface gravity waves. J. Fluid Mech., 589:433$454,2007$.

[8] D. Clamond. Note on the velocity and related fields of steady irrotational two-dimensional surface gravity waves. Philos. Trans. R. Soc. Lond. Ser. A Math. Phys. Eng. Sci., 370(1964):1572-1586, 2012.

[9] D. Clamond. New exact relations for easy recovery of steady wave profiles from bottom pressure measurements. J. Fluid Mech., 726:547-558, 2013.

[10] D. Clamond. Remarks on Bernoulli constants, gauge conditions and phase velocities in the context of water waves. App. Math. Lett., 74:114-120, 2017.

[11] D. Clamond and A. Constantin. Recovery of steady periodic wave profiles from pressure measurements at the bed. J. Fluid Mech., 714:463-475, 2013.

[12] D. Clamond and D. Dutykh. Accurate fast computation of steady two-dimensional surface gravity waves in arbitrary depth. Preprint, arxiv(247f0af79d259026338f9460aa0c8b95), 2017.

[13] D. Clamond, D. Dutykh, and A. Durán. A plethora of generalised solitary gravity-capillary water waves. J. Fluid Mech., 784:664-680, 2015.

[14] A. Constantin. The trajectories of particles in stokes waves. Invent. Math., 3:523-535, 2006.

[15] A. Constantin. Nonlinear water waves. Phil. Trans. R. Soc. A, 370(1964):1501-1504, 2012.

[16] A. Constantin. Mean velocities in a Stokes wave. Arch. Rat. Mech. Anal., 207(3):907-917, 2013.

[17] A. Constantin. The flow beneath a periodic travelling surface water wave. J. Phys. A: Math. Theor., 48:143001, 2015 .

[18] A. Constantin, W. Strauss, and E. Varvaruca. Global bifurcation of steady gravity water waves with critical layers. Acta Mathematica, 217:195-262, 2016.

[19] A. D. D. Craik. The origins of water wave theory. Ann. Rev. Fluid Mech., 36:1-28, 2004.

[20] NIST digital library of mathematical functions. http://dlmf.nist.gov/, Release 1.0.15 of 2017-06-01. F. W. J. Olver, A. B. Olde Daalhuis, D. W. Lozier, B. I. Schneider, R. F. Boisvert, C. W. Clark, B. R. Miller and B. V. Saunders, eds.

[21] W. M. Drennan, W. H. Hui, and G. Z. Tenti. Accurate calculations of Stokes water waves of large amplitude. Angew. Math. Phys., 43:367-384, 1992.

[22] W. A. B. Evans and M. J. Ford. An exact integral equation for solitary waves (with new numerical results for some 'internal' properties). Proc. R. Soc. Lond. A, 452:373-390, 1996.

[23] T. Gao, Z. Wang, and J.-M. Vanden-Broeck. On asymmetric generalized solitary gravity-capillary waves in finite depth. Proc. R. Soc. Lond. A, 472(2194):20160454, 2016. 
[24] M. S. Longuet-Higgins. Integral properties of periodic gravity waves of finite amplitude. Proc. Roy. Soc. Lond. A, 342:157-174, 1975.

[25] M. S. Longuet-Higgins. Some new relations between Stokes's coefficients in the theory of gravity waves. IMA J. Appl. Math., 22(3):261-273, 1978.

[26] M. S. Longuet-Higgins. New integral relations for gravity waves of finite amplitude. J. Fluid Mech., 149:205-215, 1984.

[27] M. S. Longuet-Higgins. Capillary-gravity waves of solitary type on deep water. J. Fluid Mech., 200:451-470, 1989.

[28] S. Murashige and S. Oishi. Numerical verification of solutions of Nekrasov's integral equation. Computing, 75(1):15-25, 2005.

[29] A. I. Nekrasov. On steady waves. Izv. Ivanovo-Voznesensk. Politekhn. In-ta, 3:52-65, 1921.

[30] I. Okamoto and M. Shōji. The mathematical theory of permanent progressive water-waves, volume 20 of Adv. Ser. Nonlin. Dyn. Worlds Scientific, 2001.

[31] I. Okamoto and M. Shōji. Trajectories of fluid particles in a periodic water wave. Philos. Trans. R. Soc. Lond. Ser. A Math. Phys. Eng. Sci., 370(1964):1661-1676, 2012.

[32] P. I. Plotnikov and J. F. Toland. The Fourier coefficients of Stokes' waves. In Nonlinear Problems in Mathematical Physics and Related Topics I, Int. Math. Ser., pages 303-315. Kluwer/Plenum, 2002.

[33] R. C. T. Rainey and M. S. Longuet-Higgins. A close one-term approximation to the highest Stokes wave on deep water. Ocean Engin., 33(14-15):2012-2024, 2006.

[34] E. R. Spielvogel. A variational principle for waves of infinite depth. Arch. Ration. Mech. Anal., 39:189205, 1970.

[35] V. T. Starr. Momentum and energy integrals for gravity waves of finite height. J. Mar. Res., 6:175-193, 1947.

[36] G. G. Stokes. On the theory of oscillatory waves. Trans. Camb. Phil. Soc., 8:441-455, 1847.

[37] G. G. Stokes. Supplement to a paper on the theory of oscillatory waves. In G.G. Stokes Math. E Phys. Papers, volume 1, pages 314-326. Cambridge University Press, 1880.

[38] W. A. Strauss. Steady water waves. Bull. Am. Math. Soc., 47:671-694, 2010.

[39] A. F. Teles Da Silva and D. H. Peregrine. Steep, steady surface waves on water of finite depth with constant vorticity. J. Fluid Mech., 195:281-302, 1988.

[40] J. F. Toland. Steady periodic hydroelastic waves. Arch. Rat. Mech. Anal., 189:325-362, 2008.

[41] M. Umeyama. Eulerian-Lagrangian analysis for particle velocities and trajectories in a pure wave motion using particle image velocimetry. Philos. Trans. R. Soc. Lond. Ser. A Math. Phys. Eng. Sci., 370(1964):1687-1702, 2012.

[42] J.-M. Vanden-Broeck. Gravity-Capillary Free-Surface Flows. Cambridge Monographs on Mechanics. Cambridge, 2010.

[43] E. Varvaruca. Bernoulli free-boundary problems in strip-like domains and a property of permanent waves on water of finite depth. Proc. Roy. Soc. Edinburgh Sect. A, 138:1345-1362, 2008.

(Didier Clamond) Université Côte D'Azur, CNRS-LJAD UMR 7351, Parc Valrose, F06108 NiCE, France.

E-mail address: didierc@unice.fr 